\title{
Analysis of Magnetic Forces in the Working Clearance with Magnetic-Abrasive Treatment of Inductors on Standing Magnets
}

\author{
A.M. Ikonnikov ${ }^{1, a^{*}}$, S.L. Leonov ${ }^{1, b}$, D.E. Solomin ${ }^{1, c}$ and A.A. Kulakov ${ }^{2, d}$ \\ ${ }^{1}$ Altai State Technical University. I.I. Polzunova, Barnaul, Russian Federation \\ ${ }^{2}$ Chenstochiv Polytechnic University, Czestochowa, Republic of Poland \\ aiamagtu@mail.ru, bsergey_and_nady@mail.ru, cd147s@mail.ru, dakulawik@icis.pcz.pl
}

Keywords: Magnetic Abrasive Treatment, Working Gap, Magnetic Abrasive Powder, Magnetic Force

\begin{abstract}
The authors describe the method of calculating the magnetic forces in the working gap in the case of magnetically abrasive machining of flat surfaces of billets from magnetic materials by the periphery of a circular inductor on permanent magnets. The application of the software package ANSIS Maxwell for the calculation of the magnetic induction method in the working gap and the magnetic forces of the magnetically abrasive powder acting on the grain is shown. As a result of the work, the magnetic induction in the working gap was calculated for magnetically abrasive machining of flat surfaces of billets from magnetic materials by an inducer on permanent magnets. Also, calculations showed the distribution of the magnetic abrasive powder in the working gap, depending on the material of the workpiece being processed. In the case of magnetically abrasive machining of a magnetic workpiece, the powder in the working gap is concentrated in the zones with the greatest density of force lines - under the inductor poles. An analysis is made of the distribution of magnetic forces in the working gap during magnetic abrasive machining.
\end{abstract}

\section{Introduction}

Magnetic abrasive machining is one of the most effective finishing operations [1]. The essence of magnetic abrasive processing is the effect on the workpiece of a powder ferromagnetic mass, compacted by the forces of a magnetic field. It is from the magnitude and distribution of the magnetic induction that the ferromagnetic mass will be distributed in one way or another in the working gap between the magnetic inductor by the workpiece surface being processed.

In magnetic abrasive processing, the role of the cutting tool is performed by a portion of the ferromagnetic powder, and the workpiece for processing is placed at a certain distance from the magnetic inductor. This space is partially or completely filled with a magnetic abrasive powder. Under the influence of the magnetic field, the grains of the ferromagnetic powder are formed into chains, forming a kind of "brush". This brush is held by the forces of the magnetic field in the working space, and when the workpiece moves relative to the inductor, it removes from the surface of the workpiece [2]. Knowing the magnitude of the magnetic forces in the working gap, it is possible to determine the location of the grains of the magnetic-abrasive powder in the working gap.

Calculation of magnetic forces in the working gap during magnetic abrasive processing It is known that the complexity of calculating the magnetic forces acting on the grains inside the working gap is aggravated by the fact that they have different shapes and sizes. It is not possible 
to take this manifold into account and it is advisable to assume that the working gap is filled with a continuous medium homogeneous in density and magnetic properties [1].

The magnetic forces acting on the grains of the ferromagnetic powder in the process of magnetic abrasive processing are calculated by the finite element method. The Ansys environment [3] will be used for this.

In this study, a planar problem is solved, and at the same time, a continuous medium homogeneous in density and magnetic properties is divided into individual grains. In this case, it is assumed that the grains of the ferromagnetic powder are ordered and have the shape of a ball.

During magnetic abrasive processing, permanent magnets are located in the inductor so that the magnetic system of the inductor consists of magnetic cells, each of which consists of a magnet and two steel magnetic circuits located at the pole side surfaces of the magnet (Fig. 1) [4].

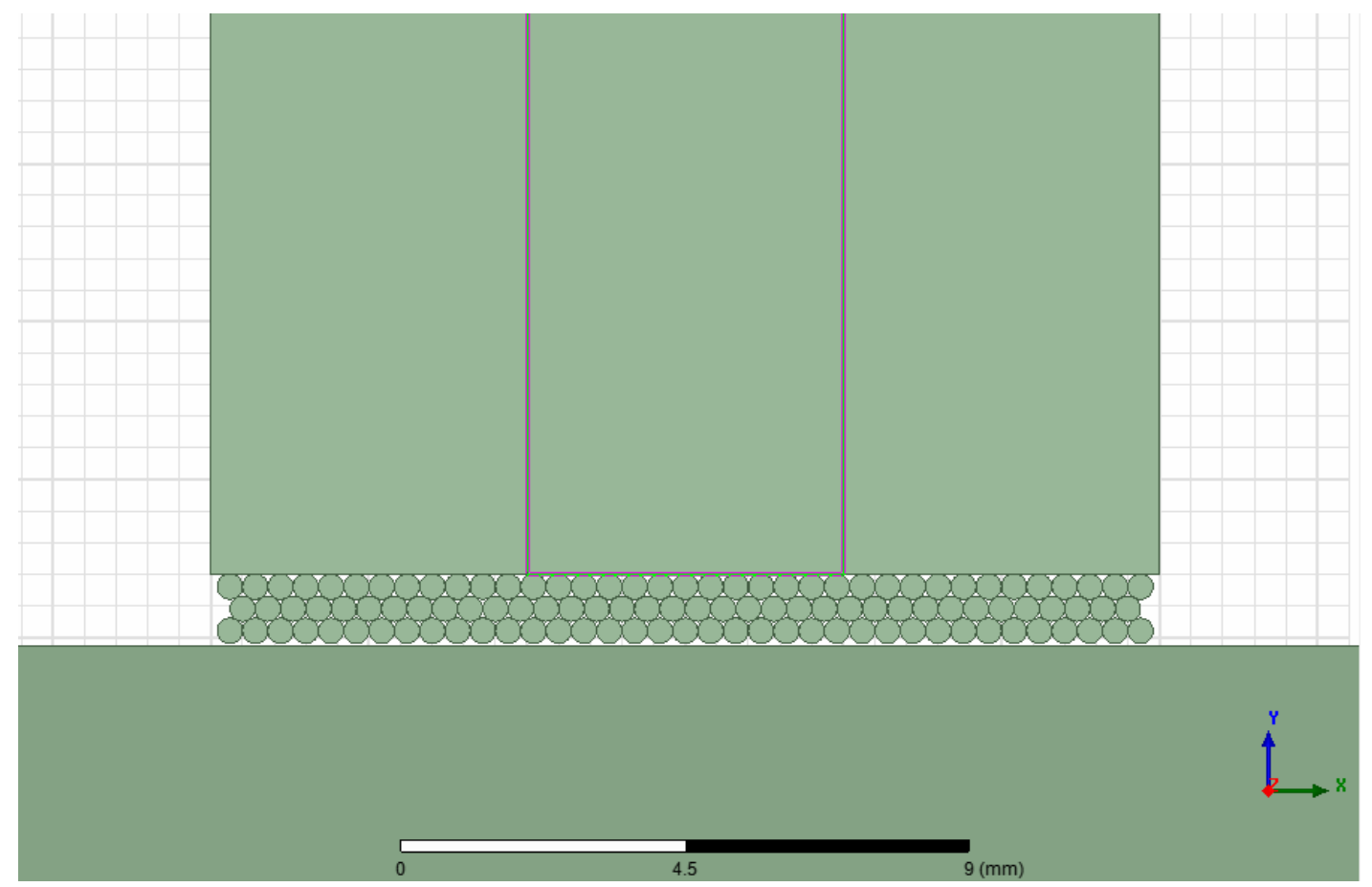

Fig. 1. Magnetic system

In this study, the calculation of the magnetic forces in the process of magnetic-abrasive processing of a workpiece from a non-magnetic material, for example, aluminum was made. It was a non-magnetic material that was taken as a sample, since it does not affect the acting magnetic forces in the working gap. When polishing a non-magnetic workpiece, the lines of force pass from one pole of the inductor to another mainly along the working gap under the magnet, concentrating at the side edges of the steel magnetic circuits (Fig. 2) [5] 


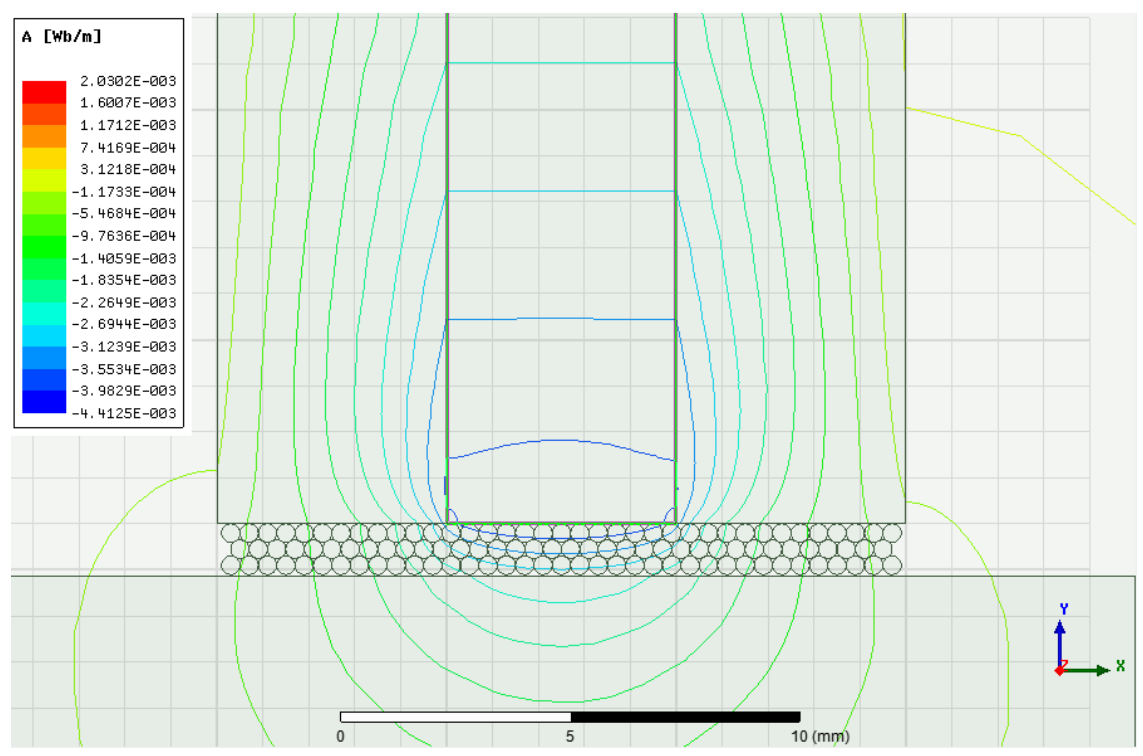

Fig. 2. Pattern of magnetic field lines

Because of this, the magnetic-abrasive powder in the working gap is concentrated under the magnets (Fig. 3).

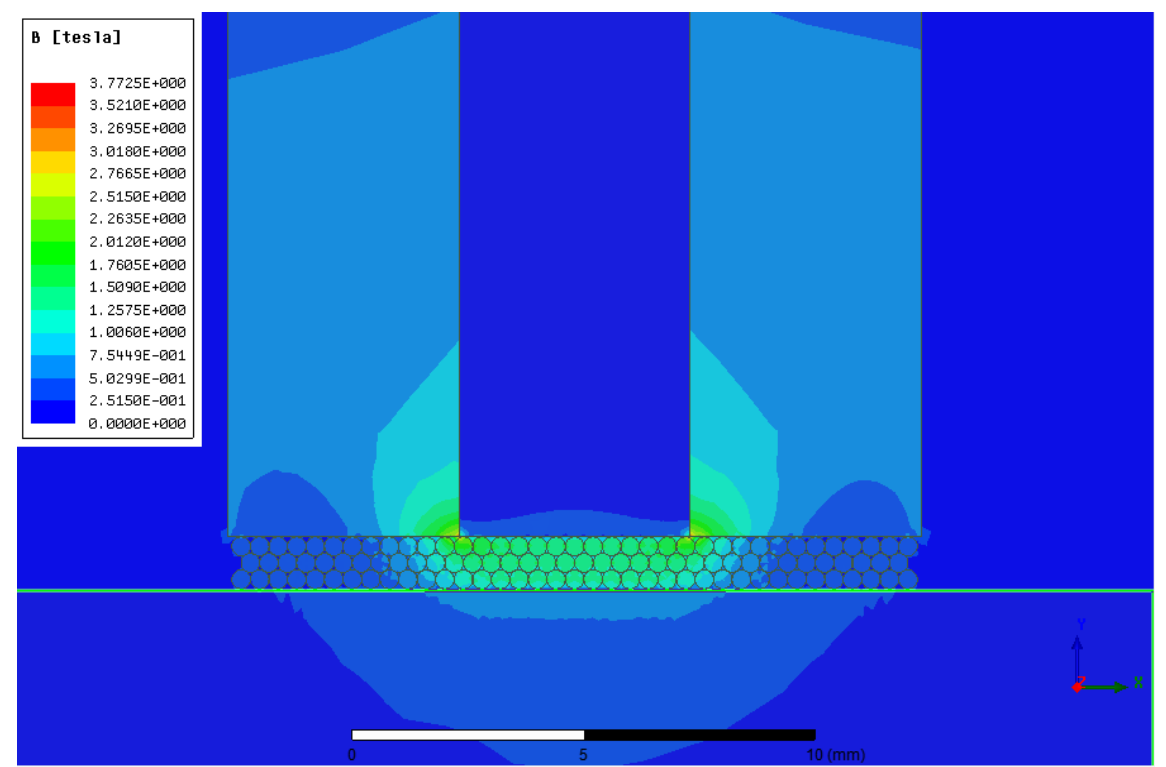

Fig. 3. Magnetic abrasive powder concentration

The next step is to calculate the magnetic forces acting on the grains of the ferromagnetic powder from the side of the magnetic field. These magnetic forces keep the powder on the working surface of the magnetic inductor [6]. Depending on the location of each specific grain in the working gap, the corresponding magnetic field force acts on the grain in a state of equilibrium.

As a result, the values of all magnetic forces acting on all grains of the ferromagnetic powder located in the working gap in a state of equilibrium were obtained [7-10]. Each grain has its own individual number, and its own value of the magnetic force. 


\section{Analysis of the magnetic forces acting in the working gap}

The graphs show the magnetic forces applied to the grains (Fig. 4).

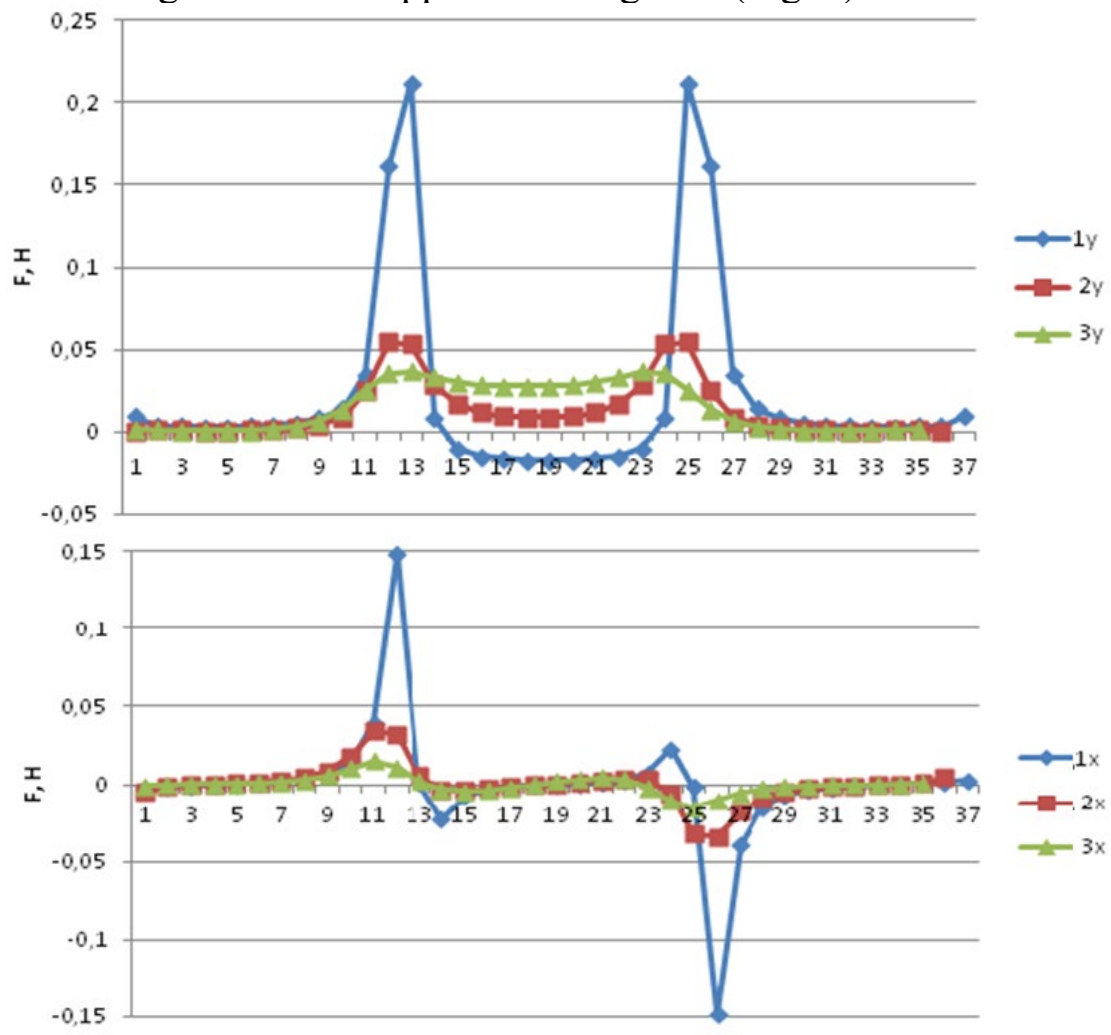

Fig. 4. Magnetic forces acting on grains

Analysis of the graphs shows that horizontal forces compress the rows of grains, ensuring their horizontal stability in the rows. This is due to the fact that for the leftmost grains the force is positive and tries to move the row to the right, and for the rightmost grains it is negative and tries to move the row to the left. The vertical force $\mathrm{P}_{\mathrm{y}}$ presses the first row of grains against the inductor (the force is positive - directed upwards), and the lower row - against the workpiece (the forces are negative - directed downward).

If we consider only 1 row of grains (at the inductor), then it is, in fact, in equilibrium: (Fig. 5, 6 ) shows the forces acting on the inductor and between the grains of the 1st row. All forces are positive - the grains are in balance. 


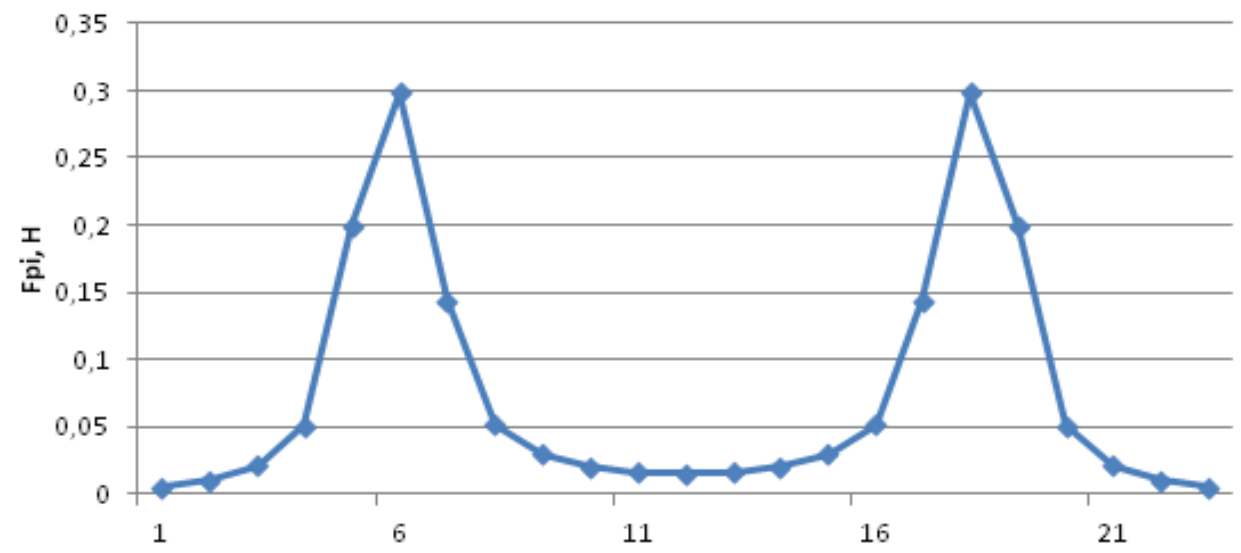

Fig. 5. The graph of forces between the grains of the first row and the inductor $F_{p i}$

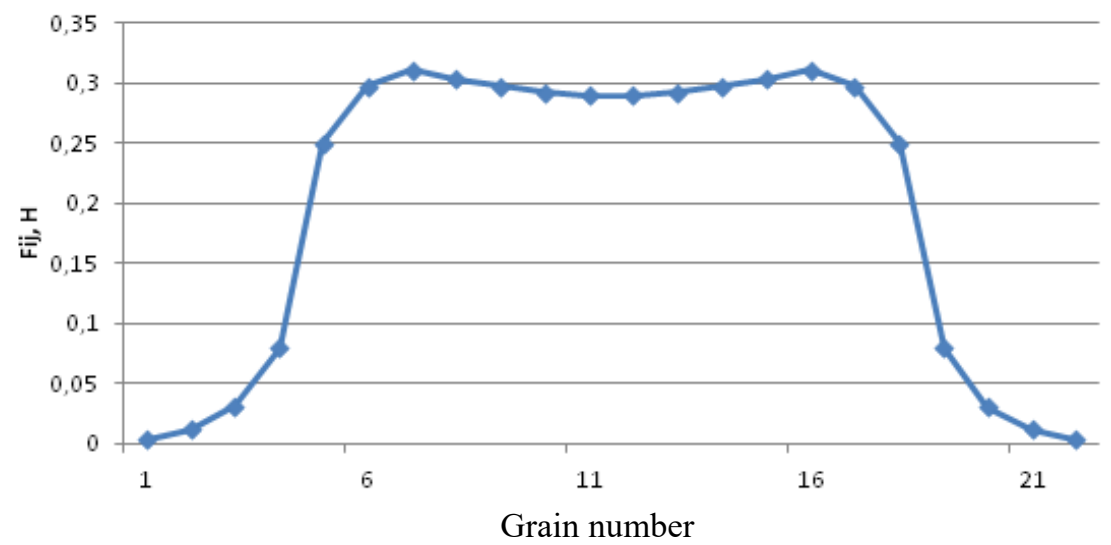

Fig. 6. Plot of forces between grains of the first row $F_{i j}$

However, if we consider the second row of grains, the equilibrium is disturbed. For the first row, equilibrium is maintained. This is due to the fact that for the extreme grains, some forces between the first and second rows have negative values. For the first row, equilibrium is maintained. If we consider all three rows, the instability becomes even greater. The instability is enhanced by the grains of the third row, which "break away" from the grains of the second row. It should be noted that the equilibrium of grains is considered without taking into account their interaction with the workpiece - cutting forces are not taken into account. With the addition of the normal components of the cutting forces acting on the grains of the third row, the stability of the grain system can be restored.

To take into account and determine the cutting forces, they must be applied to the grains of the third row, adding them with magnetic forces directed along the Y-axis - perpendicular to the workpiece surface (normal components). In this case, the maximum values of the cutting forces are determined from the condition of equilibrium of the grain system - the absence of negative forces between the grains. In fact, the cutting forces should eliminate the negative values of the contact forces between the grains of the first and second rows and the second and third rows, but not cause the appearance of negative forces between the grains of the first row. The calculation of the values of the cutting forces is reduced to their selection by the method of least squares to ensure that the forces between the grains of the first row are close to zero. The limitation is the absence 
of negative forces both in the first Rada and between the rows. Figure 7 shows the cutting forces calculated using this criterion. The curve is well approximated by the expression

$$
\mathrm{Pi}=\mathrm{A}\left[1-\exp \left(\mathrm{a}_{\mathrm{i}}+\mathrm{b}_{\mathrm{i}}^{2}\right)\right]
$$

where $\mathrm{i}$ - is the number of the third row grain.

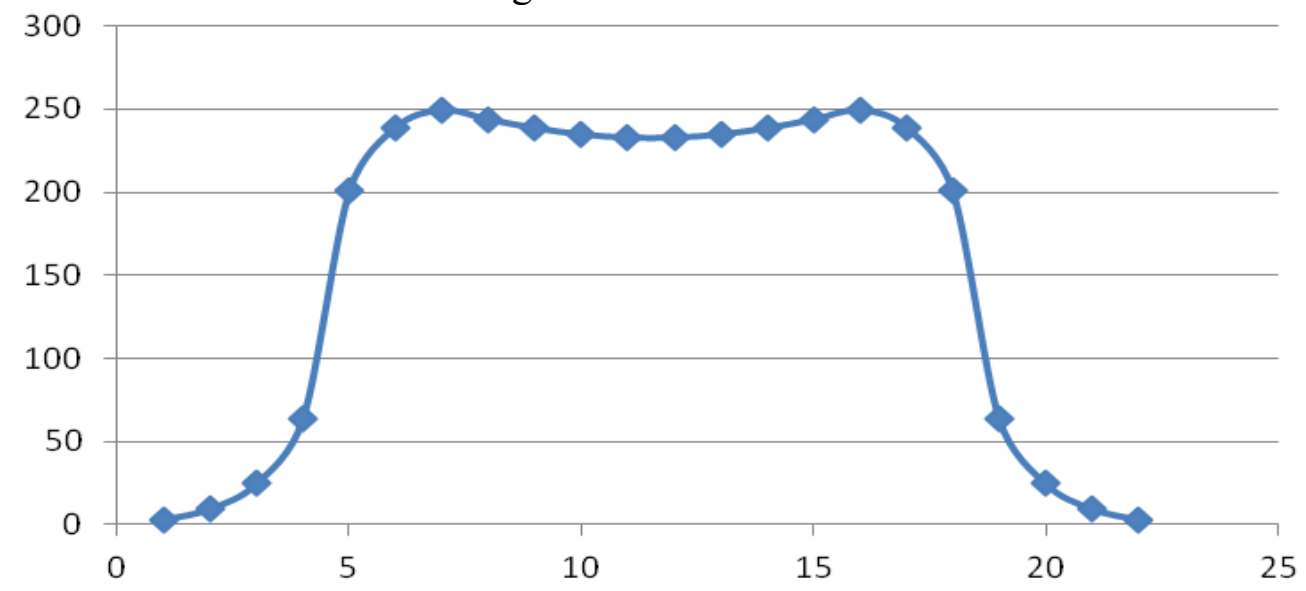

Fig. 7. Cutting forces, $N$

Due to the symmetry of the curves, the approximation was carried out for grains with numbers 1...18. The right half of the dependencies is symmetrical. Therefore, for grains 19...36, the dependence has the same form, but the numbers of grains are calculated by the formula $i=37-i_{1}$, where $i_{1}=19 \ldots 36$.

\section{Summary}

As a result of the work done, the state of equilibrium of the grains of the magnetic-abrasive powder in the process of magnetic-abrasive processing was investigated. For this, the values of the magnetic forces in the working gap have been calculated. This calculation is carried out by the finite element method, and its result is the values of all magnetic forces acting on all grains of the ferromagnetic powder located in the working gap. Knowing the magnitude of the magnetic forces, it is possible to determine the location of the grains of the magnetic-abrasive powder in a state of equilibrium. The next step is to calculate all mechanical forces in the working gap by solving the problem of static equilibrium. In contrast to the finite element method, this calculation method allows one to neglect the stresses arising from point contact of powder grains in a state of equilibrium. The result of solving this problem will be the identification of the configuration of the grains of the ferromagnetic powder in the state of static equilibrium.

\section{References}

[1] Baron, Yu. M. Magnetic-abrasive and magnetic processing of articles and cutting tools / Yu. M. Baron. - L.: Mechanical Engineering, 1986. - 176 p.

[2] Sakulevich, F.Yu. Fundamentals of magnetic abrasive processing / F. Yu. Sakulevich. Minsk: Science and Technology, 1981. - 328 p. 
[3] Prihodko, SP Simulation of the process of magnetically abrasive machining of machine parts on computers / SP Prikhod'ko // Finishing-finishing machining methods and tools in machinebuilding technology. - Barnaul, 1987. - P. 115-119.

[4] Kulavik, AA Calculation of the magnetic forces acting on the grains of a ferromagnetic powder in the process of magnetically abrasive processing / AA Kulavik, SL Leonov, AM Ikonnikov, RV Gre - benkov // Collected Works of the III All-Russian Youth Scientific and Practical School November 29- 30, 2017 "Strengthening Technologies and Functional Coatings in Mechanical Engineering", KuzGTU, Kemerovo, 2017. Access mode: http: // science .kuzstu.ru / wp-content / Events / School / utec / 2017 / school / pages / Articles / 108.pdf

[5] Prikhod'ko, SP Magnetic-abrasive polishing by inducers on permanent magnets / SP Prikhod'ko, Yu. M. Baron // Avtotorktornoeelektrooborudovanie. - 1983. - No. 5. - P. 11-14.

[6] Oliker, VE Powders for magnetically abrasive treatment of wear-resistant coatings / V.E. Oliker. - Moscow: Metallurgy, 1990. - 175 p.

[7] Leonov, SL Automatic regulation of the working gap in the case of magnetically abrasive machining of three-dimensional complex surfaces / SL Leonov, AM Ikonnikov, RV Grebenkov // Materials of the 1st International scientific-practical conference "Actual problems in machinebuilding", Novosibirsk, 2014. - P. 162-166.

[8] Prikhodko, SP The role of eddy currents in the process of magnetically abrasive processing / SP Prikhod'ko // Finishing-finishing processing methods and tools in engineering technology. Barnaul, 1989. - P. 104-107.

[9] Krymsky, M.Distribution and consolidation of a magnetic-abrasive powder in the working gap of the machine tool / MD Krymsky // Magnetic abrasive materials and methods for their testing. - Kiev, 1980. - P. 92-97.

[10] Konovalov, Ye. G. Pure machining of parts in a magnetic field by ferromagnetic powders / EG Konovalov, GS Shulev. - Minsk: Science and Technology, 1967. - 125 p. 